a Geiger counter. With each type of vessel we counted the impulses due to induced activity $(a)$ when the vessel was empty, (b) when it contained heavy water, (c) when it contained ordinary water. The results are shown in the accompanying table; the figures are the total number of counts obtained in thirty experiments for each entry. The ratio of the figures in Column $a$ is equal very approximately to the ratio of solid angles subtended in both cases.

\begin{tabular}{|c|c|c|c|c|c|}
\hline $\begin{array}{c}\text { A bsorbing } \\
\text { layer }\end{array}$ & $\begin{array}{c}\text { Empty } \\
\text { vessel } \\
(a)\end{array}$ & $\begin{array}{c}\text { Vessel with } \\
\text { heary water } \\
(b)\end{array}$ & $\begin{array}{c}\text { Vessel with } \\
\text { ordinary water } \\
(c)\end{array}$ & $b / a$ & $c / a$ \\
\hline $9 \cdot 5 \mathrm{~mm}$. & 1556 & 1898 & 4043 & $1 \cdot 22$ & $2 \cdot 6$ \\
$15 \cdot 5 \mathrm{~mm}$. & 654 & 1335 & 4173 & $2 \cdot 04$ & $6 \cdot 4$ \\
\hline
\end{tabular}

We see that the efficiency of neutrons in producing the Fermi effect is considerably increased when the neutrons pass through a comparatively thin layer of heavy water. This increase must be ascribed to the slowing down of neutrons resulting from their collisions with diplons. The effect is smaller in heavy than in ordinary water because in a head-on collision with a diplon the neutron loses only two thirds of its velocity. Calling $d_{1}$ and $h_{1}$ the coefficients by which the efficiency of neutrons is multiplied when they pass through $9 \cdot 5$ $\mathrm{mm}$. heavy and ordinary water, $d_{2}$ and $h_{2}$ the corresponding coefficients for the thickness $15.5 \mathrm{~mm}$., we see that $d_{2} / d_{2}^{2}$ is greater than $h_{2} / h_{1}^{2}$. This means that a small increase of the thickness of the scatterer corresponds to a relatively greater increase of the coefficient $d$ (heavy water) than of the coefficient $h$ (ordinary water). This difference is probably due to the fact that in the case of collisions with diplons the effective scattering takes place mostly in the backward direction, while in the case of collisions with protons the scattering is always in the forward direction. For this reason, in our arrangement multiple scattering must play a more prominent rôle in heavy than in ordinary water.

Experiments on the slowing down of neutrons in compounds of hydrogen were made by Westcott and Bjerge $^{1}$, who were led to the conclusion that the mean free path of the neutron in the scatterer decreases with their velocity and that their efficiency in silver is inversely proportional to the energy. Assuming that the cross sections of diplons and protons for collisions with neutrons are only slightly different, we find that the ideas put forward by Westcott and Bjerge account also in a satisfactory way for our experiments.

We have satisfied ourselves that the effect of heavy water described in this letter is not due to neutrons produced in heavy water by the gamma rays of radium $\mathrm{C}^{\prime 2}$, because with the small quantity of radon (40 millicuries) at our disposal, no radioactivity could be detected in silver exposed to a tube containing radon but no beryllium and surrounded by $26 \mathrm{gm}$. heavy water.

\section{H. Herszfinkiel.}

J. Rotblat.

M. Zyw.

Mirostaw Kernbaum Radiological Laboratory, Warsaw.

March 18.

1 (.. H. Westcott and T. Bjerge, Proc. Camb. Phil. Soc., 31, 145 ; 1935.

2 T. E. Banks, T. A. Chalmers and F. J. Hopwood, NATURE, 135 99, Jan. $19,1935$.

\section{Atmospheric Condensation Nuclei}

WE have measured the diffusion coefficients and the rates of fall under gravity in air of atmospheric condensation nuclei. The values obtained for the diffusion coefficients are fairly consistent, the average being about $D=18 \times 10^{-6} \mathrm{~cm} .{ }^{2} / \mathrm{sec}$. The well-known relation between mobility in an electric field and diffusion coefficient gives mobility $=D \times e N / P$. If $e$ is the electronic charge, $e N / P$ is very nearly 40 , and the corresponding mobility is $7.2 \times 10^{-4} \mathrm{~cm} . / \mathrm{sec} . /$ volt/cm., which is a probable average value for the mobility of the large atmospheric ion.

The values obtained for the rate of fall show more scatter, but observations made with two apparatus of quite different construction agree in giving a mean value of about $0.7 \times 10^{-4} \mathrm{~cm} . / \mathrm{sec}$. Comparing the rate of fall under gravity with the mobility, we have $m q \times 300 / e=0 \cdot 7 / 7 \cdot 2$. This gives for the mass of the condensation nucleus $m=1.6 \times 10^{-16} \mathrm{gram}$. If it be assumed that the nucleus is composed for the greater part of water, the radius corresponding to this mass is $3.4 \times 10^{-6}$ $\mathrm{cm}$. This is in good agreement with values derived by the application of the Stokes Cunningham law to mobility data.

Details of the experimental methods employed will be given in a later publication. From results already obtained, it appears that by these methods it will be readily possible to investigate the sizes of condensation nuclei under different conditions. The question of variation in the size of the nuclei has been the subject of much discussion, but no method of making measurements appears to have been hitherto available.

University College, J. J. NoLAN V. H. Guerrini. Dublin.

Feb. 16 .

\section{Titration Curve of Vitamin B,}

THE titration curve technique furnishes a convenient method of studying the constitution of ionisable organic compounds the possibilities of which seem to be insufficiently appreciated by many organic chemists. From one relatively simple operation, information can be obtained as to the combining weight, the number of acid and basic groups in the molecule, and their several dissociation constants. With the aid of the 'formaldehyde curve' method, it is possible to identify which of the various $p K$ values in a given complex ampholyte, acid, base or salt relate to amino, carboxyl or hydroxyl, or sulphydryl groups $^{1}$. These measurements can be done on a very small amount of material, for example, no more than a milligram or so when dealing with a substance of the molecular dimensions of vitamin $\mathrm{C}^{2}$ or $\mathrm{B}_{1}$.

Our first observations on vitamin $B_{1}$ were made with a crystalline specimen of the dihydrochloride kindly provided by Prof. B. C. P. Jansen in 1931, and more recently we have examined also specimens of a higher degree of purity obtained in 1934 through the courtesy of Prof. Jansen and of Prof. R. A. Peters. Essentially similar results were obtained on all specimens, whether derived from yeast or from rice polishings. This supports the view that the crystals represent the vitamin itself in a substantially pure state.

The vitamin dihydrochloride at dilutions around $M / 20$ in water has a $p H$ value of approximately 3 . 